\title{
Management of an Elderly Patient with Orthostatic Hypotension in Endodontic Retreatment: A Case Report
}

\author{
Michael Golden Kurniawan, Evri Kusumah Ningtyas, Bintang Adiguna Widjaja and Ira Widjiastuti \\ Department of Conservative Dentistry, Faculty of Dental Medicine, Universitas Airlangga, Surabaya, Indonesia
}

\begin{abstract}
Orthostatic hypotension occurs when cardiovascular adaptive mechanisms fail to compensate the reduction in venous return that normally occurs on the upright position. A patient with orthostatic hypotension can be a challenging case for a dentist. A 78-year-old male came with idiopatic orthostatic hypotension and felt uncomfortable with his old crown restoration and discoloration on his upper teeth. Management of orthostatic hypotension can be handled by manipulating the dental chair interval movement position.
\end{abstract}

Key Words: orthostatic hypotension, elderly patient, root canal retreatment

\section{INTRODUCTION}

Syncope is one of the most common problems that could happen to a patient in dental practice. Orthostatic hypotension, also known as postural hypotension, is the second leading cause of syncope. It appears as a result of baroreceptor reflex-mediated increase in peripheral vascular resistance failure in response to positional change. Orthostatic hypotension can be shown clinically if there is a drop in systolic blood pressure of at least $20 \mathrm{~mm} \mathrm{Hg}$ or of diastolic blood pressure of at least $10 \mathrm{~mm} \mathrm{Hg}$ within 3 minutes of standing when compared with blood pressure from sitting or supine position. ${ }^{1}$ It commonly affects older people and patients with several conditions such as neurodegenerative disease, diabetes, and hypertension. ${ }^{2}$ It is for these reasons that a dentist should be more careful in managing patients with orthostatic hypotension, especially for long and hard treatment procedures such as endodontic retreatment.

There are several causes for endodontic failures. These may be due to technical and pathological factors or systemic factors. To prevent further complications, we must first diagnose the tooth properly, after which decide whether to do root canal retreatment and restore the tooth with or without an intraradicular post and core. ${ }^{3}$

The goals in performing endodontic retreatment

Paper presented at the Joint Scientific Meeting in Special Care Dentistry, July 5, 2019, Amerta Room, 4th Floor, main campus of Universitas Airlangga, Surabaya, Indonesia.

Corresponding author: Ira Widjiastuti

Department of Conservative Dentistry

Faculty of Dental Medicine

Universitas Airlangga

J. Mayjen. Prof. Dr. Moestopo No. 47 Surabaya 60132 - Indonesia

Email: ira-w@fkg.unair.ac.id with failed primary endodontic treatment are accessing endodontic space, doing total cleaning, and disinfecting the root canal system. These can be achieved by removing various materials from the root canal such as gutta-percha, broken endodontic instruments, and sealer paste. ${ }^{4}$

One of the challenges in endodontic retreatment is the restoration part because leak of restoration like micro leakages provide opportunities for microorganisms. As we 
know most endodontically treated tooth crown is usually lost totally or partially by caries, erosion, abrasion, trauma, endodontic acces, or previous restorations. Sometimes we need to provide more retention using a root canal post where half of the coronal structure has been lost. ${ }^{5}$

Full ceramic crown is one of the best restoration choices for anterior teeth, but when combined with post and core made from metal, it often fails to give a good esthetic result due to discoloration caused by metal substructure and darkening of the gingiva. Because of this problem, a new high strength material, zirconia ceremic, is getting popular because of its opaque property that can cover the discoloration caused by metal post and core but still maintains good esthetics. ${ }^{6}$

With this perspective, the aim of this case report is to present a clinical management case to remove intraradicular post and core followed by root canal retreatment with zirconia crown and custom cast metal post-core on the right maxilary central incisor in a patient with orthostatic hypotension.

\section{CASE REPORT}

A 78-year-old male patient came to the Conservative Department of Airlangga Dental Hospital with a chief complaint of shyness and lack of confidence due to unaesthethic old crown restoration and tooth discoloration on the right maxilary central incisor teeth. On clinical

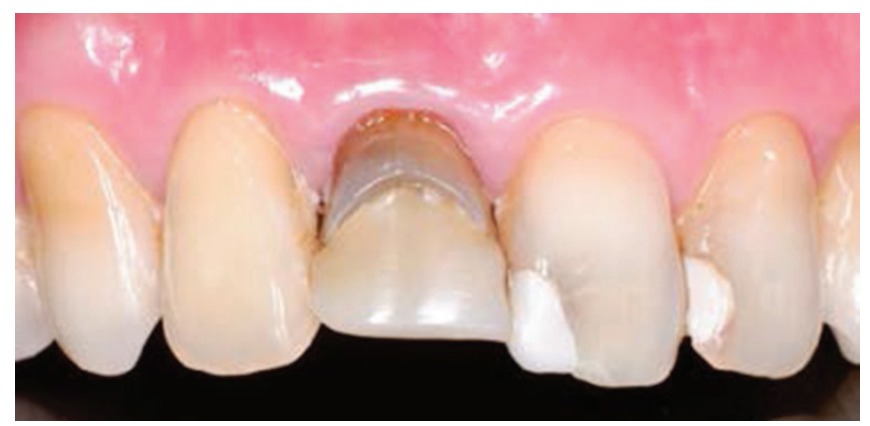

Figure 1. Preoperative clinical condition. examination, the right maxillary central incisors were found to be fractured and discolored with unesthetic old crown (Figure 1). The patient's blood pressure was $155 / 95$ and his heart rate was 90 beats/minute in the standing position. When checked in the sitting, semi-supine, and supine positions, the patient's blood pressure was $145 / 95$ and his heart rate was 80 beats/minute. Respiratory rate was 20 breaths/minute, with normal body temperature. The patient said he often feels dizzy when he stands up from the sitting or sleeping position. The patient confessed to have hypertension but is controlled and takes his medications everyday and regularly goes to his internist for his medical check up.

The patient had a history of root canal treatment in his upper front tooth, following a post and core treatment ten years prior. On radiographic examination, tooth \#11 underwent a root canal treatment but the obturation was not sealed and the working length was not achieved (Figure 2). The patient was concerned with his appearance because of his previous treatment and wanted his teeth repaired.

On the first appointment after full examination, the tooth was examined, and the patient was given a full explanation of the treatment plan, the complications that could arise, the cost of treatment, and then the informed consent.

The old restoration and the post on tooth \#11 was first removed using a crown retractor and ultrasonic system (Satelec, Acteon, North America). Obturating material were removed using peeso reamer. (Figure 3 ).

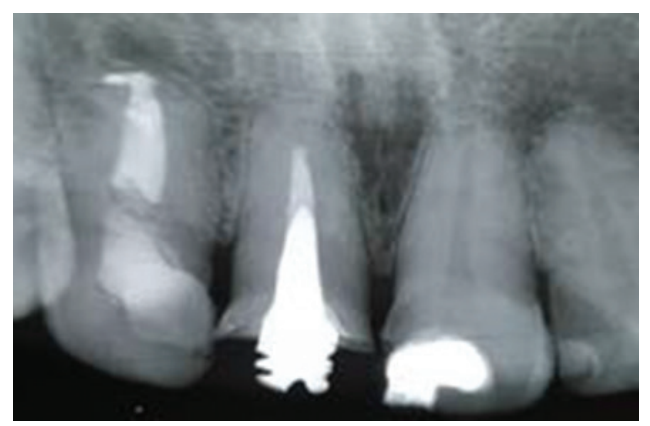

Figure 2. Preoperative intraoral periapical radiograph.
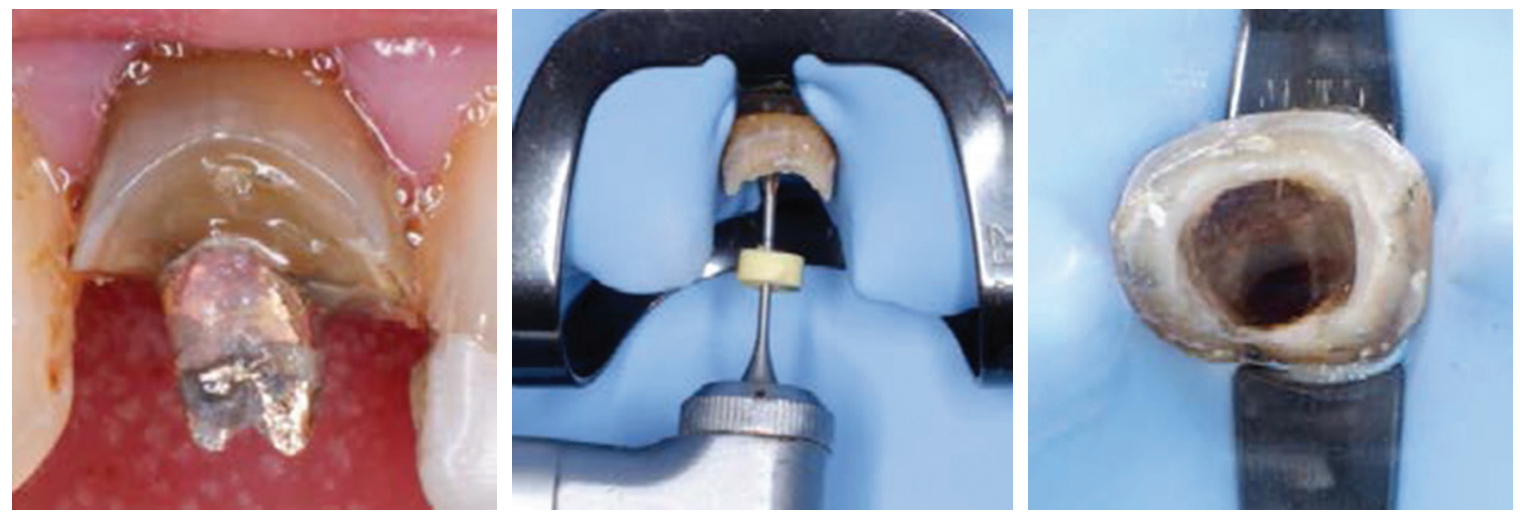

Figure 3. Removal metal post and gutta-percha. 

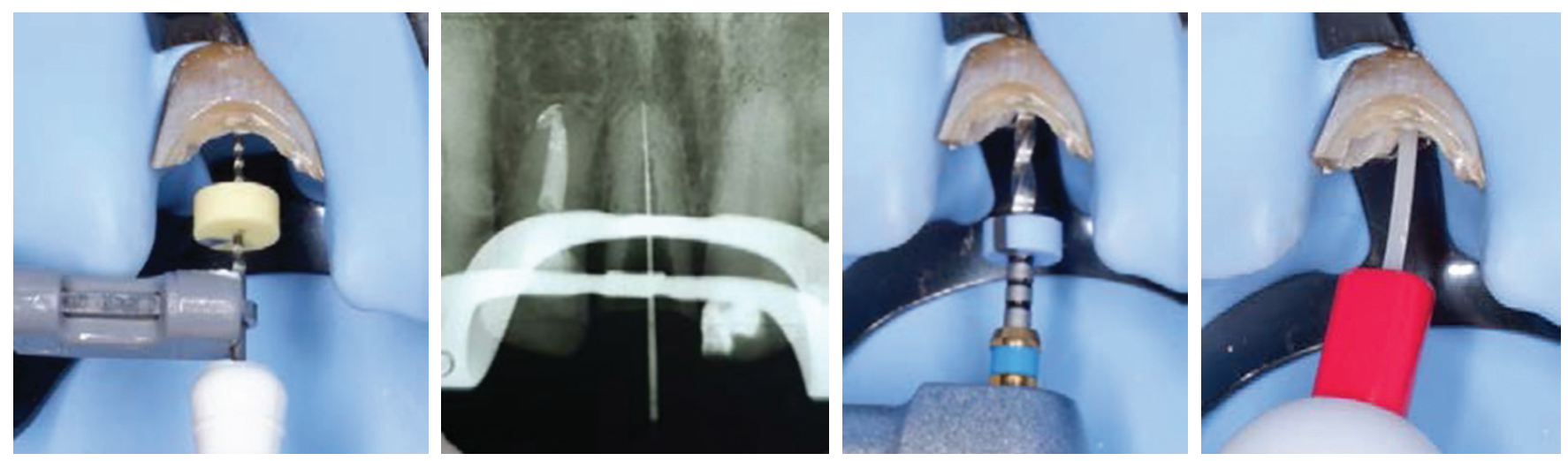

Figure 4. Procedures of root canal treatment.
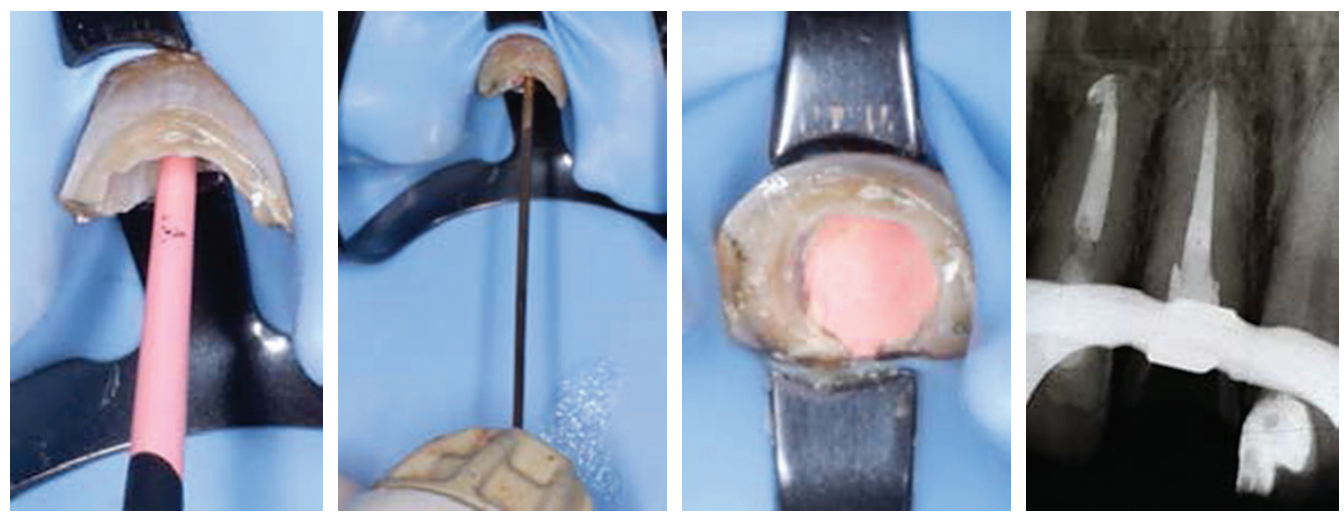

Figure 5. Obturation with thermopastic technique.
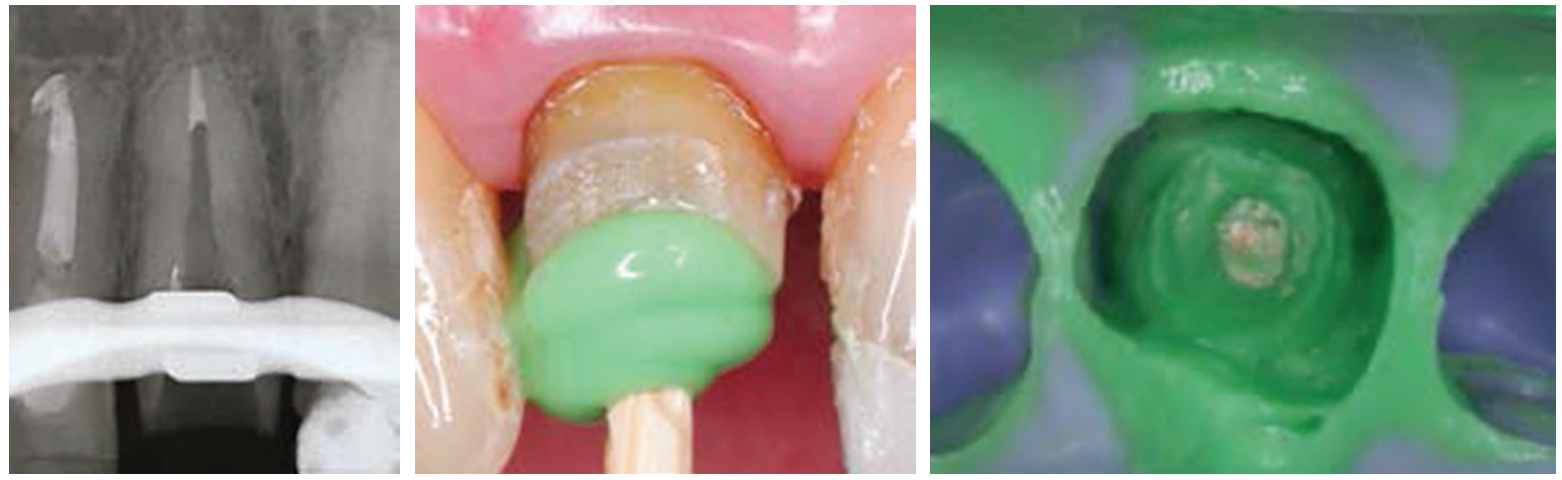

Figure 6. Impression for Custom Cast Metal Post-Core.

The working length measurement was made using an apex locator which was confirmed with a radiograph method. Preparation of the root canal was made using a Protaper Next, irrigation with $\mathrm{NaOCl} 5.25 \%$, Liquid EDTA 17\%, and Aquadest steril followed by agitation using endoactivator (Figure 4).

In the next procedure, the canal was obturated with thermopastic technique using gutta-percha master cones and resin-based sealer (Topseal, Dentsply, Konstanz, Germany) as a root canal sealer followed by gutta-percha backfill and confirmed by radiograph (Figure 5).
One week after obturation, the patient was called and the space for custom metal post core was prepared using number 2 and 3 Peeso reamers (Dentsply, Mallifier, Switzerland) maintaining $5 \mathrm{~mm}$ of gutta-percha for apical plug. Radiograph was taken to confirm the post space preparation. An impression was then made using double impression vinyl polysiloxane (Variotime, Kulzer, Germany) and hydrofilic polysiloxane (Variotime, Kulzer, Germany) for Custom Cast Metal Post-Core (Figure 6).

On the third appointment, custom cast metal postcore was inserted using luting cement (Fuji 1, GC, Japan). 

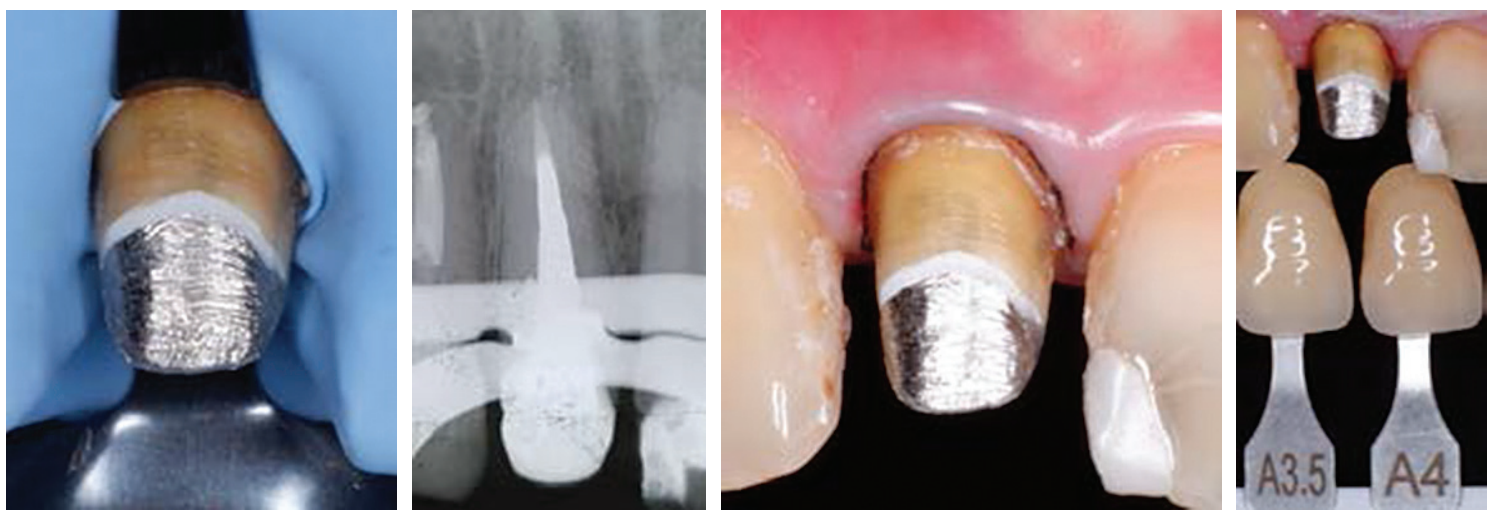

Figure 7. Custom Cast Metal Post-Core was inserted and tooth was prepared.

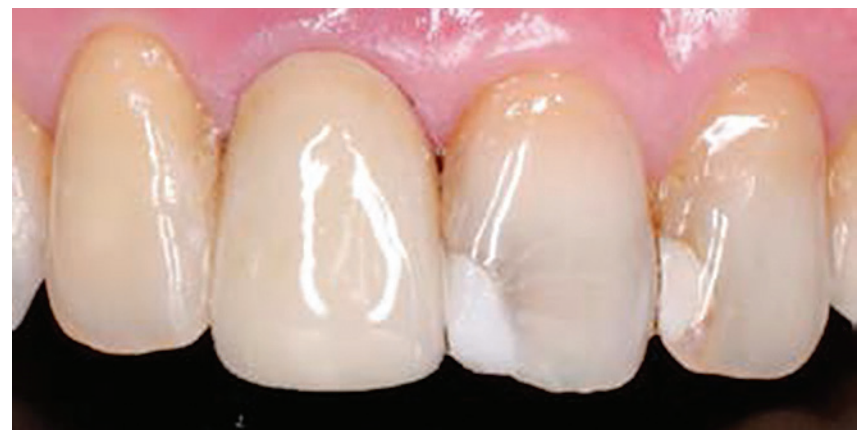

Figure 8. Clinical photo after insertion of zirconia crown on the tooth 11.

Radiograph was taken for confirmation. The tooth and the post were then prepared to make a butt joint margin finishing line. Shade selection was done using a shade guide (A-D shade guide, Ivoclar) to get a similar color-shade with the other teeth. Retraction cord was placed in the gingival sulcus for tissue management. An impression was then made with a two-step technique using vinyl polysiloxane (Variotime, Kulzer, Germany) and hydrofilic polysiloxane (Variotime, Kulzer, Germany) to restore the zirconia crown (Figure 7).

On the fourth appointment, the zirconia crown was tested and inspected for marginal fit. Lastly, the crown was inserted using luting cement (Fuji 1, GC, Japan). The excess luting agent was removed. The patient was satisfied with the result (Figure 8).

After 3 months the patient was called to evaluate the treatment outcome: the restoration was in good condition; there were no broken, cracked, discoloration found; and the condition of the supporting tissues was good with no inflammation (Figure 9).

When treating patients with orthostatic hypotension. It is important to take the patient's heart rate in the sitting, semi-supine, and supine positions. If patient's heart rate does not change and the patient does not complain of dizziness and limpness, then it is good to proceed. Changing the dental unit's position should be done slowly.

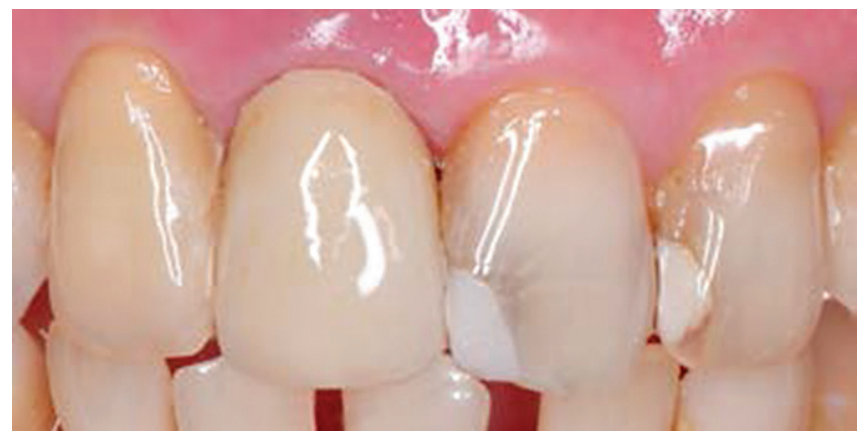

Figure 9. Clinical photo recalled patient after 3 months.

Clinicians should not change the dental unit's position in one time. The positions should be changed 2 to 3 times with a 1-minute interval to allow the patient to adapt in the new position. Blood pressure should be measured periodically if the position is changed. A clinician should stand beside a patient when the patient gets up from the dental chair unit to prevent the patient from falling because of dizziness and limpness caused by orthostatic hypotension.

\section{DISCUSSION}

Orthostatic hypotension incidents are increasing as the patient gets older. This is a major problem in aging patients. Clinician's awareness on a patient's medical history can prevent accidents due to orthostatic hypotension. Some pretreatment evaluation needs to be done before treating patients with orthostatic hypotension. These evaluations include taking the following: blood pressure, heart rate, pulse, respiratory rate, and temperature. The patient's blood pressure should be checked on several positions such as standing, sitting, semi-supine, and supine positions. A patient can be considered normal if the blood pressure test results do not surpass the $25 \mathrm{~mm} \mathrm{Hg}$ for systolic blood pressure and $10 \mathrm{~mm} \mathrm{Hg}$ for diastolic blood pressure differences; the heart rate test does not surpass 30 beats per minute between standing and supine positions. ${ }^{1}$ (Table 1 ) 
The first aid if symptoms of orthostatic hypotension develops is to allow the patient to lie down immediately. The clinician should recheck the patient's blood pressure again, if the symptoms persists. Medical consultation should then be considered before continuing with the dental treatment. ${ }^{1}$

Table 1. Clinical criteria for postural hypotension Clinical criteria for postural hypotention

Symptoms develop when individual stands

Standing pulse increases at least 30 beats per minute

Standing systolic blood pressure decreases at least $25 \mathrm{mmHG}$

Standing diastolic blood presure decreases at least $10 \mathrm{mmHG}$

The position of the dental chair unit should be changed slowly with a one-minute interval each time the position is changed to allow the patient to remain at each incremental level until dizziness dissipates. The dentist should stand close by the patient when the patient gets up from dental unit as a precaution in case the patient suddenly experiences limpness or dizziness. This is important especially in patients who undergoes dental treatments that takes long such as an endodontic retreatment. ${ }^{1}$

There are many different points of views in analyzing the need for retreatment in post-endodontically treated teeth. In European countries, studies have shown that the main reason to do root canal retreatment is periapical radiolucencies caused by failure in eliminating all the bacteria inside the root canal. The goal in doing an endodontic retreatment is to access the pulp, remove the various materials from the root canal, and to perform a good cleaning. ${ }^{3}$

Endodontically treated teeth are usually restored with posts when there is no adequate support and retention due to loss in the teeth structure. ${ }^{3}$ It should be kept in mind that the main function of a post is to build up and retain the core for crown retention, and not to strengthen the endodontically treated teeth. ${ }^{7}$

In this case, fabricated metal post was used rather than prefabricated fibre-post because it can be fitted to the root canal space. The post and core were cast as a single unit which gave a good compresive strength, the ability to modify the angulation and design of the core, the antirotational projection of the shape of the post, and extensive loss of tooth structure. ${ }^{7}$

Zirconia ceramic materials have a higer fracture resistance than other ceramics. A ceramic material with a high opacity is required when a restoration is made on an abutment such as a discolored tooth or a metal post and core. Zirconia crown is an opaque material which could be used to mask the dark colors of the underlying substructure. ${ }^{8}$ Due to its properties, zirconia was chosen as the final restoration in this case.

\section{CONCLUSION}

Patients with orthostatic hypotension can undergo long treatment times such as an endodontic retreatment procedure if the orthostatic hypotension is managed well by managing the dental chair interval movement position.

\section{Statement of Authorship}

All authors participated in data collection and analysis, and approved the final version submitted.

\section{Author Disclosure}

All authors declared no conflict of interest.

\section{Funding Source}

None.

\section{REFERENCES}

1. Malamed SF, Orr DL. Medical Emergencies in the Dental Office, 7th ed. St. Louis: Mosby, Elsevier; 2015. pp. 153-160

2. Ricci F, De Caterina R, Fedorowski A. Orthostatic hypotension: Epidemiology, prognosis, and treatment. J Am Coll Cardiol. 2015 Aug; 66(7):848-60.

3. Tomer AK, Miglani A, Chauhan P, Dubey S. Endodontic post and core retreatment - A case report. Int J Appl Res. 2017; 3(2):239-241.

4. Radeva EN. Removal of metal posts in retreatment of teeth with failed endodontic treatment (clinical cases). Int J Sci Res. 2015; 4(1):20842088.

5. Dayal C, Dhingra A, Neetika . A comparative evaluation of fracture resistance of endodontically treated teeth restored by different post system - an vitro study. IOSR Journal of Dental and Medical Sciences. 2014;13(12):63-7.

6. Vinothkumar TS, Kandaswamy D, Chanana P. CAD/CAM fabricated single-unit all-ceramic post-core-crown restoration. J Conserv Dent. 2011 Jan; 14(1):86-9.

7. Tortopidis D, Kourtis S, Kountouras K. Restoration of endodontically treated anterior teeth with cast metallic post or prefabricated fibre post placement: 2 case reports and critical literature review. Balk J Dent Med. 2015;19:86-91.

8. Suputtamongkol K, Tulapornchai C, Mamani J, Kamchatphai W, Thongpun N. Effect of the shades of background substructures on the overall color of zirconia-based all-ceramic crowns. J Adv Prosthodont. 2013 Aug; 5(3):319-325. 